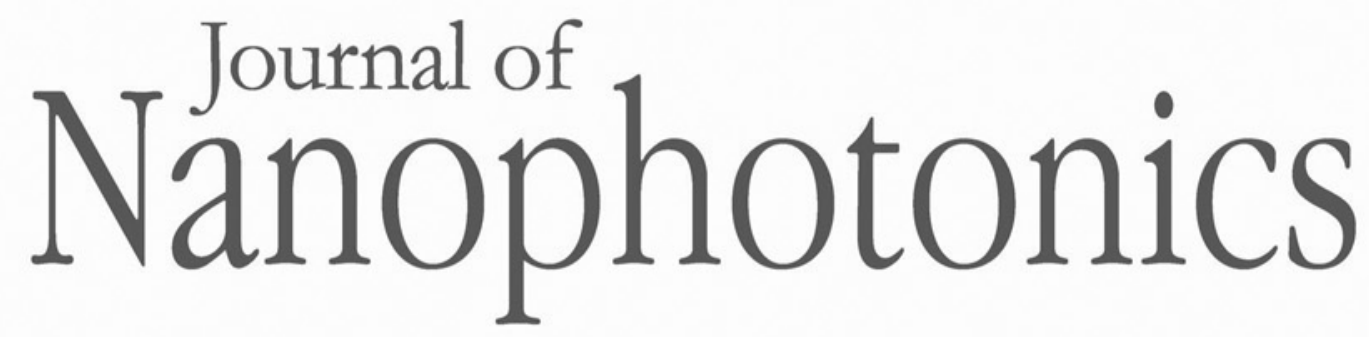

\title{
Fluorescence intensity and lifetime quenching of ceria nanoparticles as optical sensor for tiny metallic particles
}

\author{
Effat Samir \\ Nader Shehata \\ Ishac Kandas
}




\title{
Fluorescence intensity and lifetime quenching of ceria nanoparticles as optical sensor for tiny metallic particles
}

\author{
Effat Samir, ${ }^{\text {a,b }}$ Nader Shehata, ${ }^{\text {a,c,de, }}$ 网 and Ishac Kandas ${ }^{a, c, e}$ \\ ${ }^{a}$ Alexandria University, Center of Smart Nanotechnology and Photonics (CSNP), \\ SmartCI Research Center, Alexandria, Egypt \\ ${ }^{b}$ Alexandria University, Department of Electrical Engineering, \\ Faculty of Engineering, Alexandria, Egypt \\ ${ }^{c}$ Alexandria University, Department of Engineering Mathematics and Physics, \\ Faculty of Engineering, Alexandria, Egypt \\ ${ }^{\mathrm{d}}$ Utah State University, USTAR Bioinnovation Center, Logan, Utah, United States \\ ${ }^{\mathrm{e}}$ Kuwait College of Science and Technology, Doha District, Safat, Kuwait
}

\begin{abstract}
Ceria nanoparticles are studied as optical probe for different types of tiny metallic particles using fluorescence quenching technique. The synthesized ceria nanoparticles are characterized by having formed charged oxygen vacancies, which can be considered as the main receptors for the used tiny metallic particles to be sensed or absorbed. Under near-UV excitation, the visible fluorescent emission intensity is found to be reduced with increasing the concentration of the studied tiny metallic particles in an aqueous solution. To emphasize the optical sensing process, ceria nanoparticles fluorescence lifetime measurements were demonstrated before and after adding the tiny metallic particles. In addition, Stern-Volmer constants, which are considered as an indication for the sensitivity to quenchers, have been calculated for the used ceria nanoparticles and found to be 1.645 , and $0.768 \mathrm{M}^{-1}$ for both lead and iron sensing, respectively. This work could be further helpful as sensitive optical sensors in both biomedical and environmental applications. (C) 2018 Society of Photo-Optical Instrumentation Engineers (SPIE) [DOI: Q.1117/11.JNP12016007]
\end{abstract}

Keywords: ceria nanoparticles; fluorescence quenching; fluorescence lifetime; Stern-Volmer constant; particles sensors.

Paper 17144P received Sep. 22, 2017; accepted for publication Jan. 11, 2018; published online Feb. 6, 2018.

\section{Introduction}

Cerium oxide (ceria) nanoparticles extensively attract great research and commercial interests due to their observable capability to capture radicals and dissolved oxygen. 叫 $_{\text {These promising }}$ properties are useful in many applications related to the medical, environmental, and sustainable

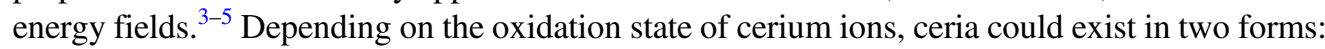
active with associated charged oxygen vacancies (O-vacancies) associated to trivalent ionization state of cerium ion $\left(\mathrm{Ce}^{3+}\right)$ and nonactive ceria with no formed $\mathrm{O}$-vacancies according to the normal cerium ion of $\left(\mathrm{Ce}^{4+}\right)$ The formed active $\left(\mathrm{Ce}^{3+}\right)$ ions undergo a relaxation process from $5 \mathrm{~d}-4 \mathrm{f}$ transitions under near-UV excitation, which results in a green wavelength fluorescence photons emission around 520-nm wavelength. The intensity peak of this fluorescence emission has a direct proportionality with the number of these O-vacancies formed during the synthesized process. Thus, these formed O-vacancies can act as optical probes to scavenge some charged particles, such as reactive oxygen species, metal ions, and dissolved oxygen. $\mathrm{G}$ In this presented research work, ceria nanoparticles have been investigated as colloidal fluorescent probe for different types of tiny metallic particles, such as lead $(\mathrm{Pb})$ particles and iron $(\mathrm{Fe})$ particles, as studied optical quenchers. Hence, any adsorption of these tiny particles in ceria

*Address all correspondence to: Nader Shehata, E-mail: hader83@vtedu

$1934-2608 / 2018 / \$ 25.00$ (C) 2018 SPIE 
O-vacancies will result in lowering the fluorescence intensity, and consequently that could be considered the sensing technique using ceria nanoparticles host. During the experimental work, different optical characterization techniques, such as absorbance dispersion, direct bandgap, and fluorescence intensity of the synthesized nanoparticles, are presented to give a proof-of-concept of having charged oxygen vacancies. Moreover, fluorescence lifetime, which is an intrinsic property, has been investigated for the synthesized ceria nanoparticles using a simple, low cost, handmade setup. In general, fluorescence lifetime measures the time by which the excited fluorophores spend in the excitation states before returning back to the ground state. ${ }^{\text {Lifetime }}$ can be considered an important characteristic that is sensitive to various parameters within ceria nanoparticles, including $\mathrm{pH}$, temperature, and oxygen vacancies concentrations, which are highly influenced after adding the studied tiny metallic particles. ceria nanoparticles as fluorescence-quenching probes for different tiny metal particles is presented in detail. The amplitude variation of the fluorescence intensity emission of the colloidal ceria nanoparticles is measured as a function of the tiny particles concentration in an aqueous solution. After that, Stern-Volmer constants are calculated at room temperature to determine the sensitivity of ceria nanoparticles toward the studied tiny particles. To emphasize the concept that ceria nanoparticles formed oxygen vacancies are the main receptors of the metallic sensing probes, fluorescence lifetime measurements have been investigated after introducing these studied tiny metallic particles.

\section{Experimental Procedure}

Ceria nanoparticles are synthesized using chemical precipitation technique, similar to what is described in Refs. 11 and 12, as it is relatively a simple procedure and needs low cost precursor. During ceria preparation, $0.5 \mathrm{~g}$ of cerium (III) chloride heptahydrate $(99.9 \%$, Sigma-Aldrich) is added to $40 \mathrm{~mL}$ deionized (DI) water, and then $1.6 \mathrm{ml}$ of ammonia is added as a catalyst. The solution is stirred over a magnetic stirrer for $24 \mathrm{~h}$ in an open container at rate $500 \mathrm{rpm}$. During the first $2 \mathrm{~h}$ of the stirring, the container is held in $50^{\circ} \mathrm{C}$ water bath and then the solution is allowed to cool to room temperature for the remaining period of stirring. To test the ability of ceria nanoparticles to attract and absorb the added tiny particles, different concentrations in grams of different types of tiny metal particles, lead $(\mathrm{Pb})$ and iron $(\mathrm{Fe})$ particles, are added to $1 \mathrm{mg} / \mathrm{ml}$ of ceria nanoparticles in DI water. Diluted solutions of ceria nanoparticles with/ without different concentrations of the studied tiny particles are inserted in quartz cuvette and optically characterized using dual-beam UV-Vis-NIR spectrometer (PG 90+ spectroscopy) that obtains the absorbance dispersion from 300 to $700 \mathrm{~nm}$ and the corresponding allowed direct band gap. The synthesized nanoparticles are then imaged using JEOL transmission electron microscope (TEM) with accelerating potential of $80 \mathrm{KV}$ while calculating the mean diameter using ImageJ software. X-ray diffraction (XRD) analysis of the synthesized nanoparticles is measured using a Rigaku XRD via $\mathrm{Cu} K \alpha$ radiation $(\lambda=+0.145 \mathrm{~nm})$. The experimental apparatus that used to correlate the fluorescence quenching mechanism for ceria nanoparticles with different concentrations of tiny metal particles is shown in Fig. 1. Tiny metal particles embedded in a diluted ceria nanoparticles solution are inserted in a fluorescence quartz cuvette that is exposed perpendicularly onto one of its sides to a 430-nm ultraviolet (UV) LED excitation. Newport cornerstone 130 monochromator is positioned perpendicularly on the other side of the quartz cuvette for collecting the obtained fluorescence intensity peak over a visible wavelength range scanned from 500 to $700 \mathrm{~nm}$ with minimum scattering. Oriel photomultiplier tube (PMT Newport 77360) is used to detect and amplify the scanned wavelength region from the monochromator output port and then send the detected data to Newport Power meter 1918R to start saving and analyzing the measured data. Within the same setup but with small modification, fluorescence lifetime measurements for ceria nanoparticles have been investigated to determine the lifetime behavior of ceria nanoparticles before and after introducing the studied tiny metallic particles. A continuous $420-\mathrm{nm}$ UV-laser source followed by a $10-\mathrm{KHz}$ optical chopper (MC2000B) is introduced as a replacement of pulsed laser source and instead of the continuous LED source in the fluorescence intensity step. These laser pulses are focused onto the cuvette that contains ceria nanoparticles after and before adding the studied tiny metallic particles. 


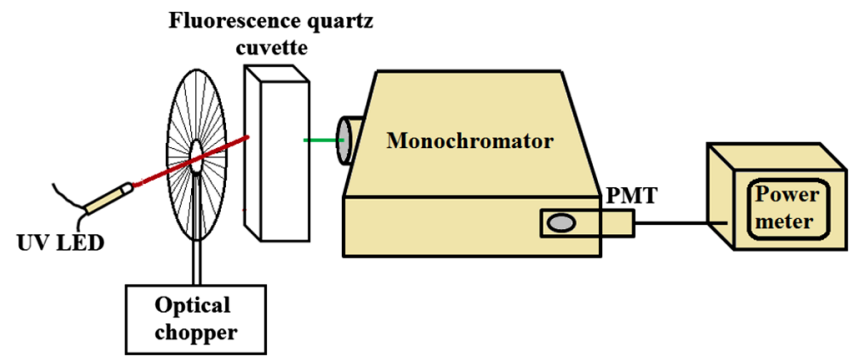

Fig. 1 Fluorescence setup used for optical sensing of tiny metal particles.

The monochromator is adjusted to collect the obtained fluorescence emission pulses from the cuvette over a fixed visible wavelength at $520 \mathrm{~nm}$. The collected pulses are detected using the PMT that is connected and followed by the power meter, which is adjusted to capture and save the emission pulses within about $5 \mathrm{~s}$ for further analysis. This simple setup fluorescence lifetime measurement setup is much lower in both complexity and cost compared to similar instruments of fluorescence lifetime spectroscopy, depending on tunable pulsed laser source as an excitation source and photon counter ${ }^{\square}$

\section{Results and Discussion}

\subsection{Characterization of Synthesized Nanoparticles}

The measured optical absorbance dispersion spectra of the synthesized nanoparticles have been shown in Fig. 2(a). The allowed direct bandgap is calculated from the linear region of the absorbance dispersion curves based on the following equation: ${ }^{[3}$

$$
\propto(E)=A\left(E-E_{g}\right)^{1 / 2}
$$

where $\alpha$ is the absorbance coefficient, $A$ is a constant that depends on the effective masses of electrons and holes in the material, $E$ is the absorbed photon energy, and $E_{g}$ is the allowed direct bandgap. The direct bandgap of the synthesized nanoparticles has been shown in Fig. 2(b). Experimentally, ceria nanoparticles bandgap is within the range from 2.7 to $3.7 \mathrm{eV}$, depending on the synthesis method, growth and postgrowth process temperatures, and the size of the particles. 1 The calculated direct bandgap of our synthesized ceria nanoparticles is closer to $3.1 \mathrm{eV}$, which indicates the existence of $\mathrm{Ce}^{3+}$ ions formation with corresponding oxygen vacancies. $4[$ Figure 2(c) shows fluorescence intensity peak of ceria nanoparticles initially without any additions, the studied tiny metal particles. The formation of visible emission peak at $520 \mathrm{~nm}$ under near-UV excitation confirms the relaxation via the $5 \mathrm{~d}-4 \mathrm{f}$ transition of excited $\mathrm{Ce}^{3+}$ ions in $\mathrm{Ce}_{2} \mathrm{O}_{3}$, which results in such a photon emission. Therefore, the higher concentration of cerium tristate ions in nonstoichiometric $\mathrm{CeO}_{x}$ along with an increase in the associated

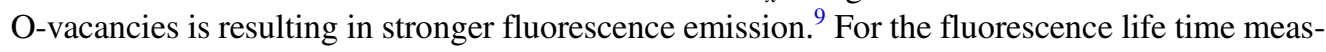
urement shown in Fig. 2(d), the duration of each emitted fluorescence pulse was about $20 \mathrm{~ms}$, where the average pulses taken in $5 \mathrm{~s}$ were obtained. Fluorescence life time values can be calculated, where the maximum intensity of the fluorescence emission is reduced by about $37 \%$ from its initial maximum value ${ }^{\mathbb{0}}$ The decaying lifetime curve is fitted to get an approximate value of the fluorescence lifetime about $5.71 \mathrm{~ms}$, which confirms that ceria nanoparticles are quite closer to be phosphorescence materials. Both TEM image and XRD pattern of the synthesized ceria nanoparticles are presented in Figs. B(a) and 3(b), respectively. The mean diameter of the nanoparticles is found to be $\sim 6 \mathrm{~nm}$. The distribution of ceria nanoparticles' size is shown in Fig. B(c), obtained from the analysis of TEM image. From the first diffraction peak of the most stable surface plane of ceria in XRD pattern, (111) plane, the mean diameter is confirmed to be $\sim 6 \mathrm{~nm}$ using Scherrer's formula [10 
Samir, Shehata, and Kandas: Fluorescence intensity and lifetime quenching of ceria nanoparticles...
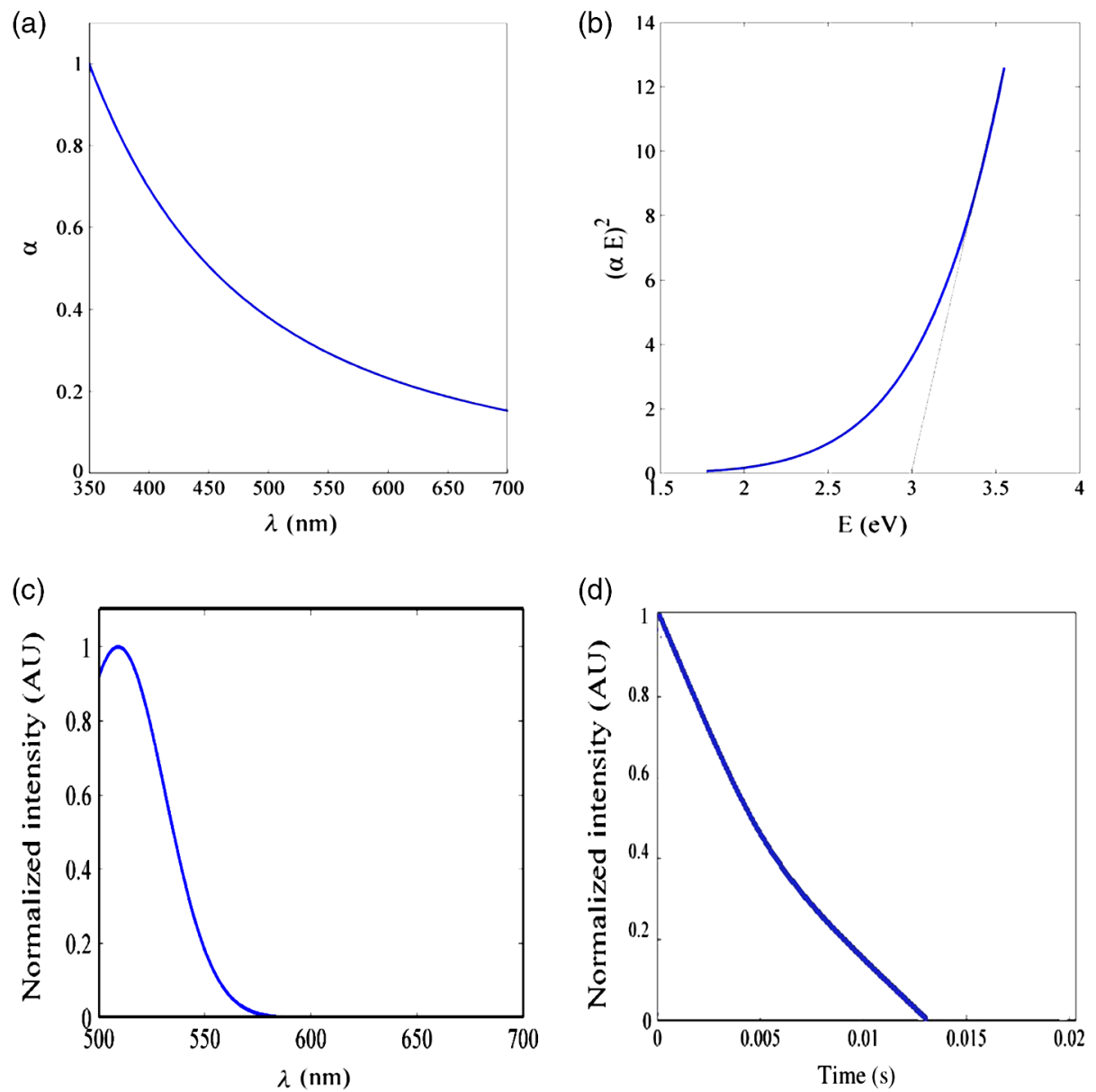

Fig. 2 (a) Absorbance dispersion, (b) direct allowed bandgap calculations, (c) fluorescence intensity, and (d) fluorescence lifetime of synthesized ceria nanoparticles.
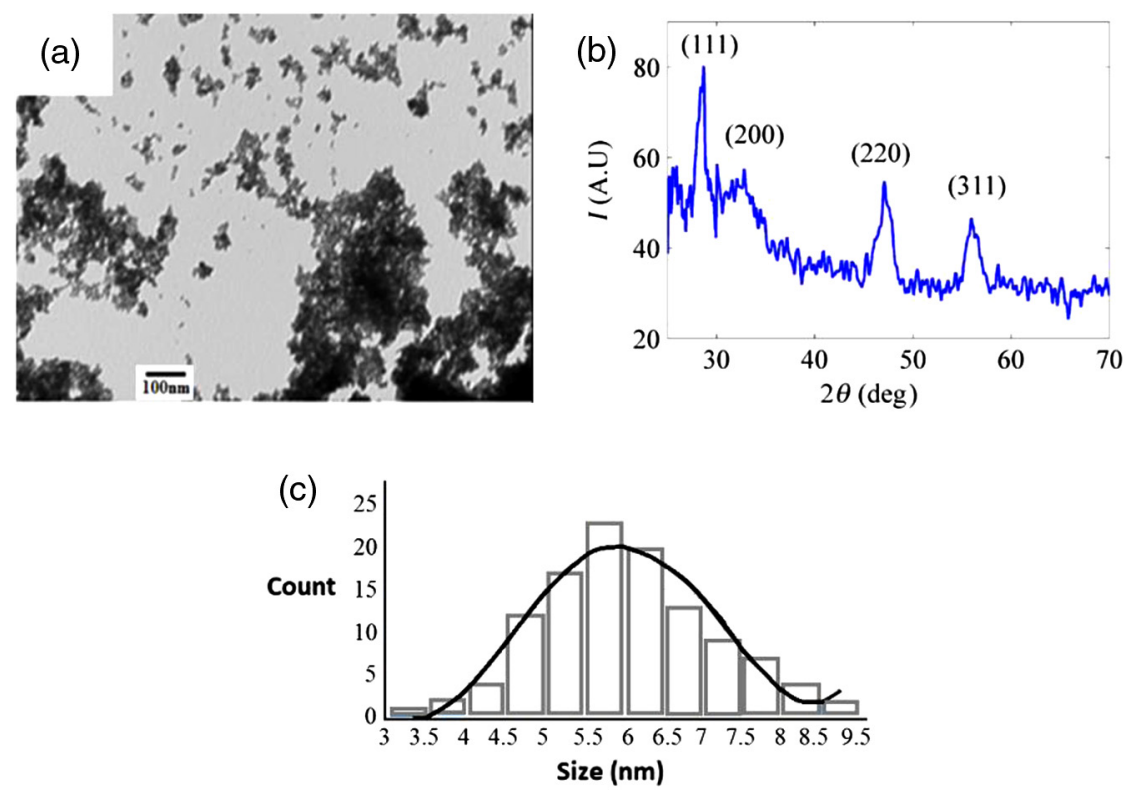

Fig. 3 (a) TEM image, (b) XRD pattern of ceria nanoparticles, and (c) size distribution of the nanoparticles. 

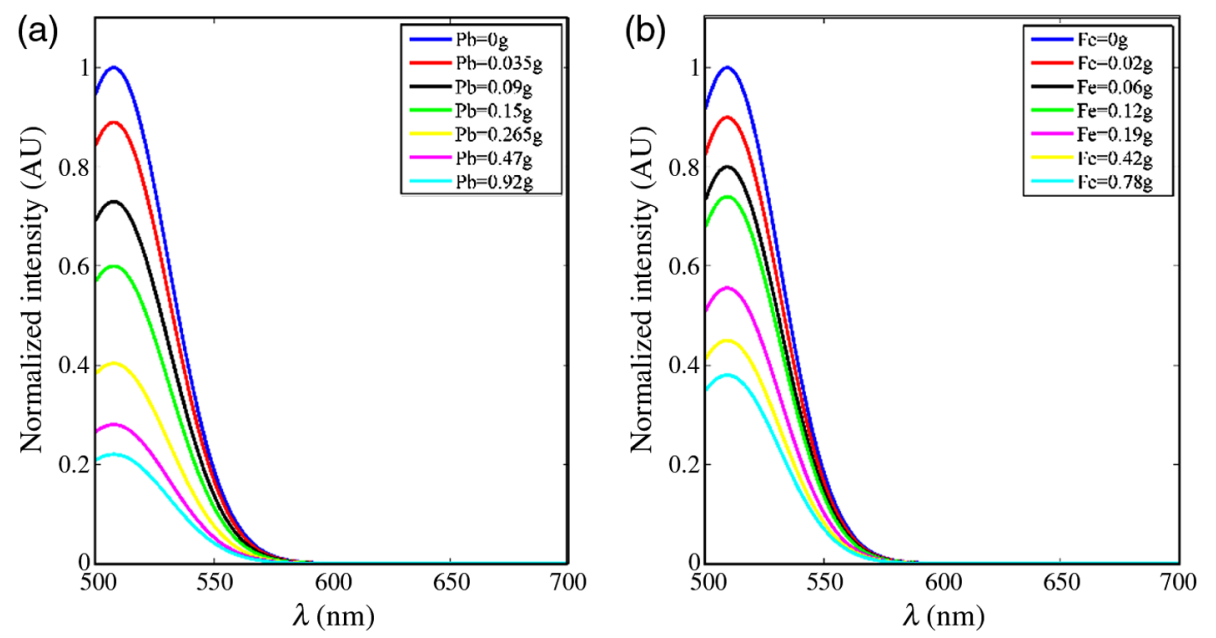

Fig. 4 Normalized fluorescence quenching due to the increase of (a) $\mathrm{Pb}$ and (b) Fe tiny metal particles concentrations at room temperature for diluted ceria nanoparticles solutions (concentration of metal particle in $\mathrm{g} / \mathrm{L}$ of the solution).

\subsection{Analysis of the Tiny Metal Particles Optical Sensing Techniques}

The visible fluorescence emissions of colloidal solutions containing $20 \mathrm{ml}$ ceria nanoparticles at different concentrations of different types of tiny metal particles, such as lead $(\mathrm{Pb})$ and iron $(\mathrm{Fe})$ tiny particles, are shown in Fig. $\theta$. Our measurements show that the synthesized ceria nanoparticles have the ability to sense the existence of very slight amounts of tiny particles. The relation between the amplitude of the fluorescence signal and the quencher concentration, tiny metal particles, is clarified in Fig. 5. The linear part only is described by the Stern-Volmer equation, as follows: 18

$$
I_{\mathrm{o}} / I=1+K_{\mathrm{SV}}[Q]
$$

where $I_{\mathrm{o}}$ and $I$ represent the peak intensities of the steady-state fluorescence in the absence and presence, respectively, of the tiny particles quenchers, $K_{\mathrm{SV}}$ is the Stern-Volmer quenching constant, which is an indication for the sensitivity of the nanoparticles to sense the existence of such quenchers, tiny particles, We found that the quenching has been saturated at concentration of $\sim 1 \mathrm{~g} / 10 \mathrm{~mL}$ of the solution. The values of the calculated $K_{\mathrm{SV}}$ values of the synthesized nanoparticles are calculated from
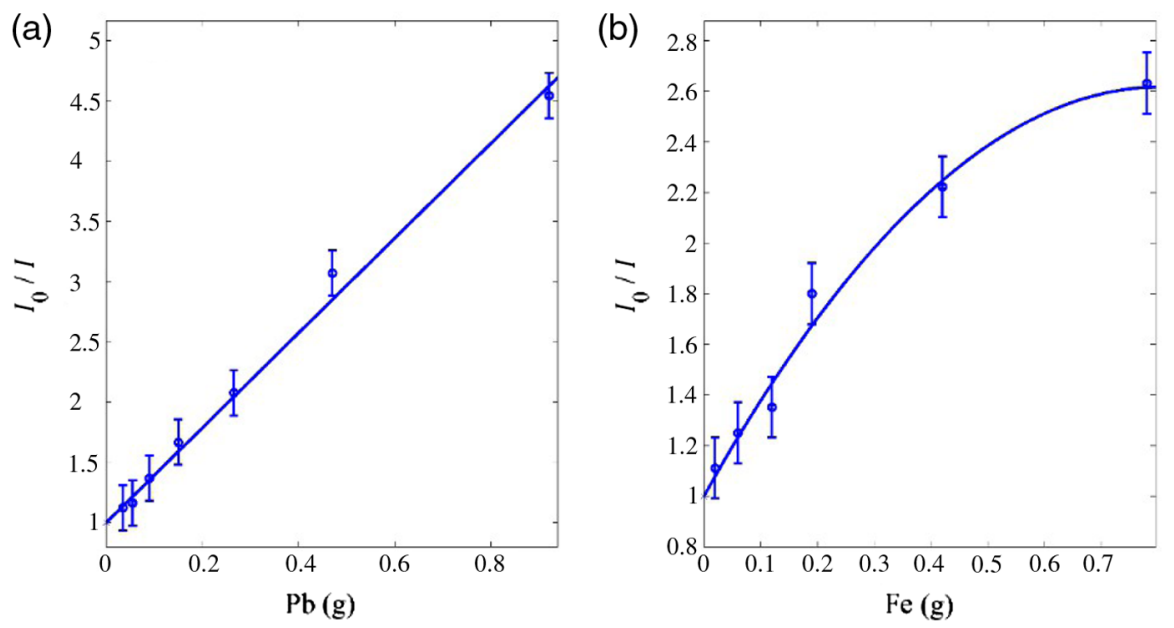

Fig. 5 Stern-Volmer relation for ceria nanoparticles solution at room temperature with different tiny metal particles concentrations: (a) Pb particles and (b) Fe particles. 
the slope of the fitted line, and found to be 1.645 , and $0.768 \mathrm{M}^{-1}$ for ceria nanoparticles toward tiny $\mathrm{Pb}$ and $\mathrm{Fe}$ particles, respectively. The relative fluorescence intensity, according to the static quenching of tiny metallic particles, shows more linear behavior in the case of lead sensing compared to iron case, which shows some saturation effect at a higher weight ratio.

Fluorescence lifetime measurements toward some chosen concentrations of the studied tiny metallic particles are shown in Fig. 6. It is clear that ceria nanoparticles fluorescence life has been suffering from a huge reduction from 5.71 to 2.48 and $2.135 \mathrm{~ms}$ after introducing up to $0.45 \mathrm{~g}$ of both tiny $\mathrm{Pb}$ and $\mathrm{Fe}$ particles, respectively, in $10-\mathrm{mL}$ solution sample. This reduction in the fluorescence life time is another confirmation that the number of the formed O-vacancies within ceria nanoparticles has been reduced after demonstrating the studied tiny metallic particles. Therefore, this could be another proof that the formed O-vacancies are the main optical receptors for the charged particles including tiny metallic particles. Hence, ceria nanoparticles are valid to be optical sensors for different types of the tiny metallic particles that exist in an aqueous solution. In both $\mathrm{Pb}$ and $\mathrm{Fe}$, the limit of detection is up to $1 \mathrm{~g} / \mathrm{L}$ of the water solution including ceria nanoparticles. The range of detection is from 0 to $1 \mathrm{~g} / \mathrm{L}$ solution. Tables 1 and $\mathrm{Z}$ show the change within ceria nanoparticles lifetime after introducing tiny $\mathrm{Pb}$ and $\mathrm{Fe}$ particles, respectively. One important point here to be mentioned that both rise and fall times of the optical pulses
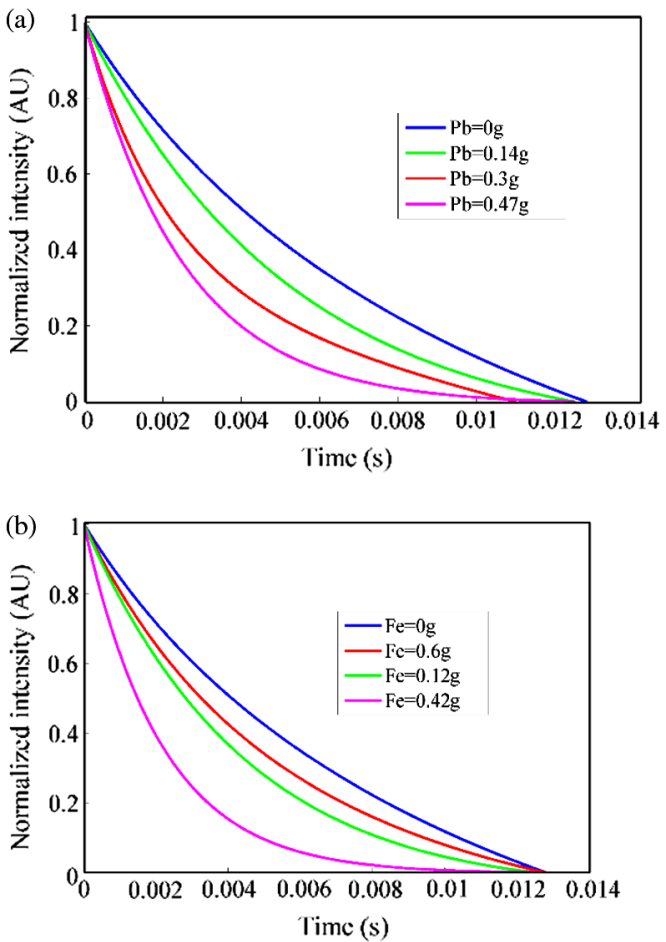

Fig. 6 Fluorescence lifetime measurements for ceria nanoparticles solution at room temperature with different tiny metallic particle concentrations: (a) Pb particles and (b) Fe particles (concentration of metal particle in $\mathrm{g} / \mathrm{L}$ of the solution).

Table 1 Fluorescence lifetime of ceria nanoparticles after adding different $\mathrm{Pb}$ concentrations.

\begin{tabular}{lc}
\hline \hline $\mathrm{Pb}$ concentration $(\mathrm{g})$ & Lifetime $(\mathrm{ms})$ \\
\hline 0 & 5.73 \\
0.14 & 4.45 \\
0.3 & 3.11 \\
0.47 & 2.48 \\
\hline \hline
\end{tabular}


Samir, Shehata, and Kandas: Fluorescence intensity and lifetime quenching of ceria nanoparticles...

Table 2 Fluorescence lifetime of ceria nanoparticles after adding different Fe concentrations.

\begin{tabular}{lc}
\hline \hline Fe concentration $(\mathrm{g})$ & Lifetime $(\mathrm{ms})$ \\
\hline 0 & 5.73 \\
0.06 & 4.63 \\
0.12 & 3.98 \\
0.42 & 2.13 \\
\hline \hline
\end{tabular}
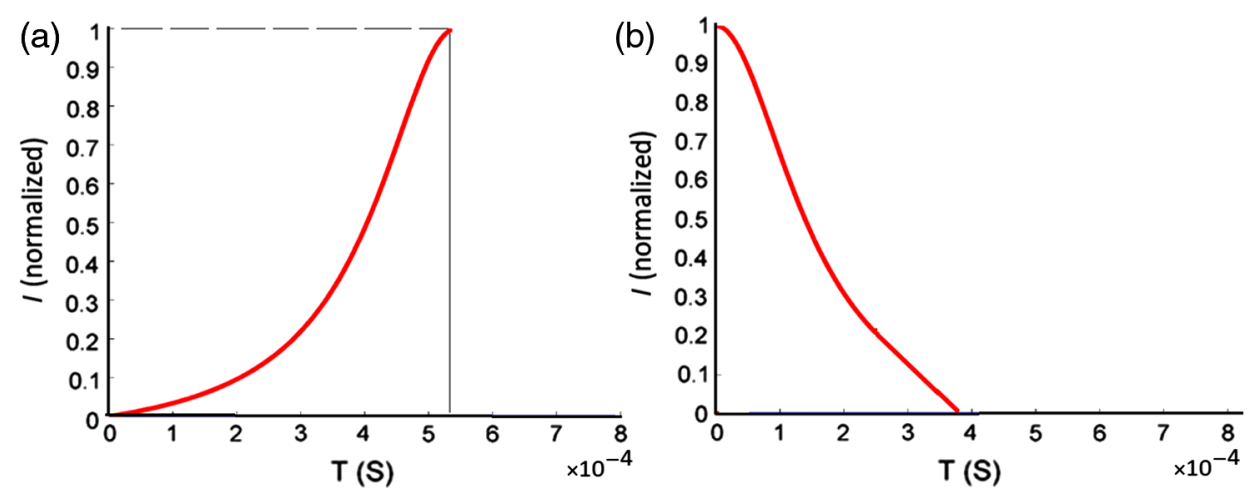

Fig. 7 (a) Rise and (b) decay curves of optical excitation due to the optical chopper rotation at $10 \mathrm{KHz}$.

generated from the optical chopper at $10 \mathrm{KHz}$ are shown in Fig. 7. It is observed that both rise and fall times do not exceed $0.55 \mathrm{~ms}$, which is much smaller compared to the obtained fluorescence lifetime. This confirms the fact that the fluorescence lifetime experimentally detected has low contribution of the setup response and the dominant impact is coming from the fluorescence itself.

\section{Conclusions}

The presented work introduces a new application of the synthesized ceria nanoparticles as colloidal sensors for different types of tiny metallic particles sensing with fluorescence quenching technique. Our synthesized ceria nanoparticles are characterized to prove its capacity of $\mathrm{O}$-vacancies, which could be the probes for tiny particle ions, such as lead $(\mathrm{Pb})$ and iron $(\mathrm{Fe})$. Ceria nanoparticles have reduced visible fluorescence emission peaks with increasing the tiny metallic particle concentrations under 430-nm optical excitation. Stern-Volmer constants are calculated to determine ceria nanoparticles sensitivities toward the tiny metallic particles, which show that ceria nanoparticles are highly sensitive toward such quenchers. Another proof that ceria nanoparticles of O-vacancies are the main receptors for the existence of the tiny metallic particles, fluorescence lifetime measurements have been demonstrated using a simple, low cost, handmade setup. Fluorescence lifetime value has been reduced also after introducing the studied tiny metallic particles. This work could be helpful in some biomedical, industrial, and environmental applications.

\section{Acknowledgments}

The authors appreciate the financial support of Smart Critical Infrastructure (SmartCI) in Alexandria University for supplying some facilities used in the prepared fluorescence spectroscopy. 


\section{References}

1. M. Quaranta, S. M. Borisov, and I. Klimant, "Indicators for optical oxygen sensors," Bioanal. Rev. 4, 115-157 (2012).

2. N. Feng, J. Xie, and D. Zhang, "Synthesis, characterization, photophysical and oxygensensing properties of a novel europium(III) complex," Spectrochim. Part A 77, 292-296 (2010).

3. B. A. Rzigalinski et al., "Radical nanomedicine," Nanomedicine 1, 399-412 (2006).

4. N. Shehata et al., "Nano-enriched and autonomous sensing framework for dissolved oxygen," Sensors 15, 20193-20203 (2015).

5. N. Shehata et al., "Enhanced erbium-doped ceria nanostructure coating to improve solar cell performance," Materials 8, 7663-7672 (2015).

6. T. Skala et al., "Formation of alumina-ceria mixed oxide in model systems," Appl. Surf. Sci. 257, 3682-3687 (2011).

7. N. Shehata et al., "Reduced erbium-doped ceria nanoparticles: one nano-host applicable for simultaneous optical down- and up-conversions," Nanoscale Res. Lett. 9, 231 (2014).

8. D. Chorvat and A. Chorvatova, "Multi-wavelength fluorescence lifetime spectroscopy: a new approach to the study of endogenous fluorescence in living cells and tissues," Lasers Phys. Lett. 6, 175-193 (2009).

9. J. R. Lakowicz, Principles of Fluorescence Spectroscopy, 3rd ed., Kluwer/Plenum, New York (2006).

10. L. Marcu, "Fluorescence lifetime techniques in medical applications," Ann. Biomed. Eng. 40(2), 304-331 (2012).

11. N. Shehata, K. Meehan, and N. Jain, "Control of oxygen vacancies and $\mathrm{Ce}^{+3}$ concentrations in doped ceria nanoparticles via the selection of lanthanide element," t. Nanopart. Res. 14, 1173-1183 (2012).

12. H. Chen and H. Chang, "Homogeneous precipitation of cerium dioxide nanoparticles in alcohol/water mixed solvents," Colloids Surf. A 242, 61-69 (2004).

13. J. Pankove, Optical Processes in Semiconductors, 1st ed., Dover Publications Inc., New York (1971).

14. L. Yin et al., "Sonochemical synthesis of cerium oxide nanoparticles-effect of additives and quantum size effect," T. Colloid Interface Sci. 246(1), 78-84 (2002).

15. V. Ivanov et al., "Formation of nanocrystalline ceria from cerium(III) nitrate solutions in aqueous alcohol," Dokl. Chem. 411, 223-225 (2006).

16. A. Shmyreva, A. Borisov, and N. Maksimchuk, "Electronic sensors built on nanostructured cerium oxide films," Nanotechnol. Russ. 5, 382-389 (2010).

17. S. Deshpande et al., "Size dependency variation in lattice parameter and valency states in nanocrystalline cerium oxide," Appl. Phys. Lett. 87, 133113 (2005).

18. B. Cullity, Elements of X-Ray Diffraction, 1st ed., Addison-Wesley Publishing Company Inc., Massachusetts (1956).

19. C. Chu and Y. Lo, "A plastic optical fiber sensor for the dual sensing of temperature and oxygen," EEEE Photonics Technol. Lett. 20, 63-65 (2008).

20. M. Iosin, V. Canpean, and S. Astilean, "Spectroscopic studies on $\mathrm{pH}$ - and thermally induced conformational changes of bovine serum albumin adsorbed onto gold nanoparticles," T. Photochem. Photobiol. A 217, 395-401 (2011).

Biographies for the authors are not available. 